\title{
Evaluación de las estrategias de ingreso de Colombia al Foro de Cooperación Económica Asia-Pacífico (APEC)
}

\section{Evaluation of Colombian Income Strategies at the Asia Pacific Economic Cooperation Forum APEC}

\author{
págs. $45-54$ \\ Grupo de Investigación: Desarrollo y Equidad \\ Línea de investigación: Desarrollo económico, sectorial e industrial \\ Henry Laverde Rojas• Luz Rocío Corredor González••
}

Recibido: agosto 01 de 2016

\section{RESUMEN}

Cada país prioriza objetivos de corto, mediano y largo plazo para la instrumentalización de sus estrategias políticas y económicas de inserción internacional. Colombia no ha sido ajena a esta dinámica. Luego de establecer prioridades y objetivos, que están articulados a los objetivos e instrumentos de su política comercial, la política económica colombiana ha buscado implementar acuerdos comerciales, tratados de libre comercio, acuerdos de asociación económica, etc. Uno de los objetivos del país es ingresar al Foro de Cooperación Económica Asia-Pacífico (APEC), que es una instancia superior de su proceso de integración económica internacional; sin embargo, no lo ha logrado por la falta de impacto en las estrategias de inserción y, por falta de institucionalidad y seguimiento a la implementación de la misma. De eso trata el artículo, de explorar por qué no ha sido posible ingresar a APEC, meta que se considera prioritaria dentro de la política exterior colombiana.

Palabras clave: política exterior, cooperación, geoeconomía, China, Colombia.
Aceptado: noviembre 25 de 2016
Given that the foreign policy of countries and international relations themselves are dynamic, each country prioritizes short-, medium- and long-term objectives that are instrumented in its International Insertion Strategy. Colombia has not been alien to this dynamic, and after establishing such priorities and objectives for the achievement of it and articulated to the objectives and instruments of trade policy, as a tool of the Colombian Economic Policy translated into Trade Agreements, Free Trade Agreements Trade, Economic Partnership Agreements, etc. It has sought to enter the Asia Pacific Economic Cooperation Forum APEC, as a higher instance of its integration process, where it prizes mutual benefit over individual interests, however, it has not succeeded because of lack of assertiveness in the Strategy of integration or perhaps because of lack of institutionality and follow-up in its implementation. That is what the article is about, to explore because it has not been possible that achievement that is considered a priority within the Colombian Foreign Policy

Key words: foreign policy, cooperation, China, Colombia, geoeconomy

\footnotetext{
-Ph. D. en Economía. Docente investigador, Facultad de Economía, Fundación Universidad América. henry.laverde@profesores.uamerica edu.co

-• Economista, Docente Investigador, Facultad de Economía, Fundación Universidad de América. Iuz.corredor@profesores. uamerica.edu.co
} 


\section{INTRODUCCIÓN}

Acorde con la evolución de su política exterior y las nuevas dinámicas de las relaciones internacionales, Colombia ha implementado una estrategia de inserción internacional que contempla objetivos de corto, mediano y largo plazo. En términos de tiempo, los objetivos de corto plazo buscan identificar prioridades de relacionamiento que se ajusten a las expectativas del país para integrarse al mundo e incluyan, en algunos momentos, el componente geográfico, es decir, ir de continente a continente para articularse a las metas de la política comercial internacional. Al respecto, hay que considerar que la política exterior y la política comercial forman parte de la política económica del país e inciden en su crecimiento económico y desarrollo.

En ese contexto, desde principios del siglo XXI, todas las prioridades del país han girado alrededor de la resolución del conflicto armado, fenómeno que se ha identificado como la causa de los bajos niveles de crecimiento económico y de las problemáticas sociales y políticas del país. Entre 2002 y $2010^{1}$ se articuló una política exterior que estuvo anclada a la política de "seguridad democrática", bandera política del ex presidente Álvaro Uribe Vélez en sus dos períodos de gobierno. Según esta política, si el Estado garantizaba la seguridad de los ciudadanos dentro del territorio colombiano, todo lo demás vendría por añadidura; además, esta política de seguridad proponía una resolución militar y armada del conflicto, por lo que se incrementó el gasto en defensa y armamento. Se sacrificaron, entonces, todos los recursos -incluyendo los de cooperación internacional-para el logro de este objetivo, en detrimento de los indicadores sociales y con implicaciones directas sobre la población, sobre todo de las áreas rurales en las regiones (Peña, 2014).

A partir de 2010, el discurso cambia con la presidencia de Juan Manuel Santos, que estructura la política nacional alrededor de la importancia de tener una paz negociada, por lo que se propuso buscar el respaldo de la comunidad internacional. Paralelamente, este gobierno aceleró la implementación de una política comercial basada, entre otros instrumentos, en el incremento de la entrada en vigencia, negociación y aprovechamiento de los acuerdos comerciales suscritos por Colombia (Hamburger, 2014).

La articulación de la política económica internacional con el tema de paz y los derechos humanos, así como con los de mecanismos para incrementar los niveles de competitividad y de inversión extranjera directa, generaron una sinergia que permitió exportar más y en mejores condiciones (con todo lo que esto implica para lo económico, social e, incluso, ambiental), lo que ha puesto a Colombia en un nuevo escenario geoestratégico, donde China, como integrante del Foro de Cooperación Económica Asia-Pacífico (APEC), tiene un atractivo especial y desempeña un rol determinante.

La estrategia de inserción internacional, como instrumento de la política exterior y comercial de Colombia, representa un reto que ha ido materializándose en objetivos de corto, mediano y largo plazo desde lo geográfico, político, económico y social. Sin embargo, a pesar de haberse priorizado el ingreso del país al APEC, que es un paso importante en el proceso de integración, aún no se ha logrado; por lo que el acercamiento a China, como potencia global, es un eje estratégico que está pendiente en la agenda internacional de Colombia.

1 Según algunos analistas e internacionalistas (ver, por ejemplo, Ardila, 2005), en este periodo no hubo una política exterior. 
LÍNEA DE INVESTIGACIÓN: DESARROLLO ECONÓMICO, SECTORIAL E INDUSTRIAL

Este balance propone una lectura sobre la dinámica de la política exterior colombiana y su articulación a la política comercial del siglo XXI como estrategia de inserción internacional, así como analiza lo que significa APEC y China (Ardila, 2005) para esta estrategia.

\section{MÉTODO}

Aunque algunos internacionalistas lo niegan (ver, por ejemplo, Ardila, 2005), sí puede afirmarse que durante las dos primeras décadas del siglo XXI existió en Colombia una política exterior y de relaciones internacionales. En la primera década, esta política se caracterizó, fundamentalmente, por priorizar en la idea de una salida militar al conflicto armado, lo que redujo la política exterior a una diplomacia catalítica (Ripoll de Castro, 2015), que buscó el respaldo de la comunidad internacional como alternativa para garantizar la seguridad a los ciudadanos, apoyándose, entre otros, en los recursos de cooperación internacional, por lo que se implementó el Consenso de Londres y el Consenso de Cartagena.

Sin embargo, en lo corrido de la segunda década, el discurso ha cambiado, así como las prioridades de la política exterior. Ya no se relaciona la búsqueda de recursos y respaldo internacional para construir una política exterior belicista, sino que la política exterior busca, con el acompañamiento de la comunidad internacional y los organismos internacionales (Ramírez, 2011), poner fin a la guerra y dar paso a una política exterior más constructivista (Merke, 2007), que se apoye en los constructos sociales de la experiencia propia e internacional para dar paso a una sociedad moderna e incluyente (Hernández, 2009).

En este periodo la política exterior colombiana es más moderna y dinámica, y el Estado tiene un margen más amplio de acción y la facultad de realizar alianzas estratégicas de integración y cooperación con nuevos actores, así como asumir nuevos retos y ampliar la agenda en pro de sus propios intereses. Dicha política está asociada a un proceso de integración que buscó, en una primera etapa, acceder al mercado de los Estados Unidos con un tratado de libre comercio para demostrar que el país contaba con las condiciones para competir en los mercados internacionales (se negociaron otros tratados que complementaron este propósito, como los suscritos con Chile o Canadá). La segunda etapa de la integración fue la firma del acuerdo comercial con la Unión Europea, que buscó posicionar productos agrícolas (orgánicos, principalmente) e implementar el cumplimiento de normas y estándares internacionales (estas normas buscan que el sector agrícola se consolide y que se garantice su trazabilidad y acceso a recursos financieros y técnicos, así como a la transferencia de nuevas tecnologías limpias). De igual manera, el ingreso de Colombia a la Organización para la Cooperación y el Desarrollo Económicos (OECD), club de buenas prácticas, avala al país como un socio confiable desde lo económico y lo político.

El tercer gran momento proyectado es el ingreso de Colombia al mercado de Asia-Pacífico, por considerarse un escenario de integración que busca complementariedad y beneficio mutuo, lo que constituye un reto de mediano plazo y que tendría impactos de largo aliento; propósito que el presidente ha ratificado en varias oportunidades, como en la reciente cumbre de APEC, realizada en Lima (Perú), en la que recibió el respaldo total de sus miembros al proceso de paz (APEC, 2016).

Adicionalmente, es importante aclarar que la estrategia de inserción internacional implica la instrumentalización de las acciones que conducen al logro de los objetivos de corto, mediano y largo plazo, para que el Estado colombiano interactúe con otros Estados en el sistema internacional, con 
el fin de: identificar oportunidades para la oferta exportable del país, atraer inversión extranjera y ser sujeto de recursos de cooperación (a pesar de ser un país de renta media). Es decir, la estrategia de inserción internacional implica: a) aprovechar las ventajas y beneficios de los otros países con los que Colombia va a relacionarse y b) posicionar a Colombia ante los otros países según sus potencialidades y las necesidades de los otros países. Igualmente, entre otras opciones, el país puede buscar un liderazgo regional que multiplique buenas prácticas de cooperación (Tassara, 2016).

Estos procesos conllevan unos costos y beneficios políticos que, si son administrados eficiente y asertivamente, pueden tener altos impactos en el papel que el país desempeña en los distintos escenarios internacionales y más allá de lo meramente económico. El APEC es uno de ellos y actualmente está tomando cada vez más protagonismo en el concierto internacional, porque este mecanismo de cooperación propone el beneficio mutuo y potencial entre los miembros, en términos de flujos de comercio, consumo, inversión extranjera, ingreso per cápita, entre otros.

No obstante las debilidades del país, constituir alianzas con países como China puede facilitar el ingreso de manera bilateral al APEC, lo que no ha podido lograrse de manera multilateral (Torres, 2014).

\section{RESULTADOS}

Dentro de su estrategia de inserción internacional, Colombia ha identificado unas prioridades, definidas como objetivos en el paso del tiempo, que han estado relacionadas con expectativas y necesidades comerciales, en unos casos, y con perspectivas políticas, en otros. Esto hace que la estrategia tenga fuertes elementos de política comercial, a los que hay que sumar a otros elementos en los que se combina lo económico, lo social, la cooperación y el beneficio mutuo, como es el propósito de APEC.

Si bien Colombia no ha sido aceptada como miembro de APEC (el país presentó su primera solicitó formal de ingreso en 1996), participa como observador en grupos de trabajo relacionados con energía, telecomunicaciones e información y promoción comercial. Actualmente participa en las reuniones de trabajo del grupo de expertos en inversión en el grupo de energía y se espera que más adelante pueda ingresar nuevamente al grupo de telecomunicaciones (Pérez y Pérez, 2011). Estas estrategias de participación e involucramiento no han tenido un significado importante, porque, por un lado, no son aprovechadas suficientemente en términos técnicos y de participación de expertos y, por otro, no tienen una trascendencia política ni decisoria.

De acuerdo con investigadores expertos en el tema de Asia-Pacífico (Pérez y Pérez, 2011), para lograr que Colombia se convierta en miembro de APEC es necesario mejorar aspectos a nivel interno, como infraestructura portuaria, presencia diplomática y comercial permanente de Colombia en Asia, así como negociar la firma e implementación de acuerdos comerciales con miembros de la región que faciliten el aumento de sus exportaciones.

Además, Colombia debe conocer e involucrarse en la dinámica de APEC, lo que implica reconocer que algunos desafíos externos no dependen directamente del país. Frente a las economías de ASEAN +5 (la cual excluye a la India), las exportaciones de Colombia a la región solo representan el $8,7 \%$ del total de las exportaciones al mundo, en donde China es el principal destino (Villa, 2015). En contraste, las exportaciones de Chile y Perú a la región tienen un porcentaje de $43.8 \%$ y 
LÍNEA DE INVESTIGACIÓN: DESARROLLO ECONÓMICO, SECTORIAL E INDUSTRIAL

24.5\%, respectivamente; y si bien para México las exportaciones a China solo representan el $3 \%$, en cantidad de productos sobrepasan las exportaciones de Colombia. China es uno de los principales destinos comerciales de México, Chile y Perú (Villa, 2015).

Las estrategias implementadas por Colombia buscan adaptarse a la dinámica de APEC, lo que incluye trabajar en el dilema de la expansión, la definición de la agenda de sus reuniones, la nueva arquitectura del sistema asiático y la proliferación de acuerdos de libre comercio entre las economías miembros de APEC. No obstante, existen factores externos que están más allá de la política exterior colombiana, en términos de moratoria para ingreso de nuevos miembros, así como la exigencia de desarrollar una agenda paralela que le permita cumplir con los requisitos fijados en la Cumbre de Bogor de 1994 (Indonesia), relacionados con liberalización del comercio, las inversiones y la facilitación de los negocios (Tassara, 2016).

Recientemente, en 2011, se constituyó la Alianza del Pacífico con el objetivo de acelerar el ingreso de Colombia en APEC (Tremolada, 2015). De los países fundadores de esta iniciativa de integración regional (Chile, México, Perú y Colombia), únicamente Colombia no pertenece al Foro de Cooperación Económica Asia-Pacífico (Sánchez, 2015). Esta estrategia, quizás por ser la más reciente, ha permitido que Colombia gane espacios en el liderazgo regional y sea reconocida como potencia media en temas relacionados con la paz; aunque esto aún debe aprovecharse más y generar impactos más fuertes que lleguen hasta APEC. Es importante que Colombia actúe como la voz de los que no tienen voz en este lado del Pacífico, que sea su vocero y contribuya a extender las relaciones entre el Pacífico oriental y Pacífico occidental.

Colombia no puede desconocer la importancia de pertenecer a APEC, no solo por su ubicación geoestratégica, sino por ser este el principal mecanismo de cooperación económica del Pacífico asiático. Además, pertenecer a este mecanismo de cooperación permitiría diversificar el comercio, incrementar la inversión y facilitar el acceso del país a nuevas tecnologías. En esta medida el Estado colombiano debe diseñar estrategias que le permitan establecer metas y articular espacios y mecanismos que garanticen el ingreso de Colombia APEC; entre estos mecanismos se encuentran: mejorar la infraestructura portuaria, aumentar la presencia institucional en Asia, incrementar los tratados de libre comercio con los miembros de APEC y motivar al sector privado para que mejore el intercambio con la región y trabaje con el sector público y la academia para articular un proceso coherente y consistente de inserción económica en esta región (Pérez y Pérez, 2011).

El ingreso de Colombia a APEC, tiene muchos impactos positivos, no sólo para el país en su evidente interés por consolidar su proceso de integración, sino para América Latina como mercado objetivo de Asia Pacífico, que puede aprovecharse para fomentar la cohesión social a través de la cooperación económica, pero también el potenciamiento de la oferta exportable y de nuevos sectores (como el ambiental) que promuevan el desarrollo sostenible (Piedrahita, 2015).

El acercamiento entre China y Colombia es evidente, las expectativas mutuas son palpables, casi tangibles (Vidal, Peláez y Miranda 2015), al igual que las necesidades y los mecanismos en que pueden complementarse económicamente, lo cual implica riesgos y costos, pero también oportunidades y nuevas posibilidades para sectores emergentes de Colombia en China y el Asia Pacífico y viceversa.

Muchos estudios pretenden encontrar respuestas para la integración China-Colombia (Mateus, 2015). Sin embargo, la clave está en construir los perfiles de los dos países desde distintas ópticas, incluyendo lo cultural y el modelo de negociación que utiliza cada uno, para no solo identificar 
los intereses económicos potenciales de las dos partes, sino también la propia evolución de sus procesos de integración y las sus motivaciones y pretensiones. La cooperación económica busca el beneficio mutuo y la complementación de sus matrices de intereses, así como, quizás implícitamente, nuevas formas de poder como mecanismos de soft power (Lamus, 2015); mecanismos que hay que descifrarlos, no para superarlos, sino para desarrollar instrumentos nuevos e innovadores que posibiliten el óptimo, eficaz y eficiente uso del espacio de integración propuesto, para que la competitividad no solo sea para las empresas y los sectores económicos, sino para los propios Estados y sus maneras de administrar y gestionar las formas de poder emergentes de la contemporaneidad.

En la tabla 1 se evalúa en cifras de 2010 la relación entre Colombia y Asia y entre China y Colombia en cifras (precios US FOB)

Tabla 1

Balanza comercial Colombia-Asia 2010

\begin{tabular}{lrrc}
\hline & Exportaciones & Importaciones & Balanza comercial \\
\hline Asia & 2.853 .705 .056 & 6.982 .075 .117 & -4.128 .370 .061 \\
Japón & 512.521 .472 & 1.086 .977 .122 & -574.455 .649 \\
China & 1.968 .151 .744 & 5.027 .630 .015 & -3.059 .478 .271 \\
Corea del Sur & 373.031 .839 & 867.467 .980 & -494.436 .140 \\
\hline
\end{tabular}

Fuente: Ministerio de Comercio, Industria y Turismo (2016).

\section{DISCUSIÓN}

El Foro de Cooperación Económica Asia Pacífico (APEC) es, sin lugar a dudas, un espacio de integración regional que ha trascendido las fronteras para convertirse en un ejemplo de lo que puede hacerse más allá de las pretensiones económicas, y del que muchos quieren ser parte por su contexto político, económico e ideológico. China es el eje comercial y político de APEC, no solo por la magnitud de su economía y su demografía, sino por su manera de hacer las cosas, porque creó una integración que estaba más allá de la conveniencia coyuntural y creó un proyecto de largo aliento que permitirá compensar los costos y los riesgos y motivar el proceso de construcción de una visión prospectiva de la integración.

Colombia ha realizado esfuerzos ingentes por ingresar a APEC, a tal punto que constituyó la Alianza del Pacífico como una estrategia intermedia y estableció un bloque comercial conformado por cuatro países de América Latina que son parte de APEC y que apoyan el ingreso de Colombia. Dicha alianza, que se formalizó con la declaración de Lima del 28 de abril de 2011, quiere construir una agenda donde quepan todos los temas que promuevan el comercio exterior de los países miembros y de los observadores, para generar mayores índices de crecimiento económico y, a la vez, acercarse a APEC, así como lo evidencian las estadísticas (que son, sin lugar a dudas, en el principal respaldo a dicho ingreso). 
Tabla 2

Importaciones China-Colombia

\begin{tabular}{|c|c|c|c|c|c|c|}
\hline $\begin{array}{c}\text { Impor } \\
\text { de china } \\
\text { en miles } \\
\text { de U\$ }\end{array}$ & & $\begin{array}{c}\text { Apa y } \\
\text { material } \\
\text { eléctrico, } \\
\text { de } \\
\text { grabación } \\
\text { o imagen }\end{array}$ & $\begin{array}{c}\text { Reactores } \\
\text { nucleares, } \\
\text { calderas, } \\
\text { máquinas y } \\
\text { partes }\end{array}$ & $\begin{array}{c}\text { Vehículos } \\
\text { automóviles } \\
\text { tractores } \\
\text { ciclos } \\
\text { partes y } \\
\text { accesorios }\end{array}$ & $\begin{array}{c}\text { Manufactura } \\
\text { de } \\
\text { fundición, } \\
\text { de hierro o } \\
\text { acero }\end{array}$ & $\begin{array}{r}\text { Calza } \\
\text { botin } \\
\text { artícı } \\
\text { análog } \\
\text { part }\end{array}$ \\
\hline 2015 & U\$1.727 & U\$485 & U\$343 & U\$61 & U\$48 & \\
\hline 2014 & U\$ 1.790 & U\$3.196 & U\$2.429 & U\$504 & U\$381 & $U$ \\
\hline 2013 & U\$10.632 & U\$2.751 & U\$2.173 & U\$411 & U\$342 & $u$ \\
\hline
\end{tabular}

Fuente: tabla elaborada con base en los datos de Ruíz (20 de mayo de 2016 
Tabla 3

Exportaciones colombianas a China

\begin{tabular}{lrrr}
\hline \multicolumn{1}{c}{ Producto } & \multicolumn{1}{c}{$\mathbf{2 0 1 3}$} & \multicolumn{1}{c}{$\mathbf{2 0 1 4}$} & \multicolumn{1}{c}{$\mathbf{2 0 1 5}$} \\
\hline Aceites Crudos de Petróleo & $4.296 .869,35$ & $5.198 .852,49$ & $1.796 .758,38$ \\
Ferroníquel & $325.526,07$ & $182.251,58$ & $212.802,41$ \\
Desperdicios y desechos de Cobre & $230.409,63$ & $159.924,87$ & $134.574,32$ \\
Hullas Bituminosas & $65.929,33$ & $75.054,68$ & $14.335,72$ \\
Cueros y pieles, curtidos & $25.550,61$ & $20.412,67$ & $13.529,53$ \\
Insecticidas, raticidas & $12.395,43$ & $10.049,97$ & $12.731,04$ \\
Alcohol de petróleo & $24.024,89$ & $29.151,58$ & $12.251,12$ \\
Desperdicios y desechos de aluminio & $38.996,39$ & $12.550,00$ & $11.132,55$ \\
Cueros y Pieles enteras & $18.536,34$ & $7.072,00$ & $10.224,93$ \\
Café sin tostar & $3.840,01$ & $5.679,88$ & $7.698,34$ \\
Otros & $61.884,44$ & $54.135,08$ & $37.686,46$ \\
\hline Total & $5.103 .962,49$ & $5.755 .134,80$ & $2.263 .724,80$ \\
\hline
\end{tabular}

Nota: Los valores se encuentran en miles de dólares.

Fuente: DANE (2016)

Quizás, a simple vista, las exportaciones no representan un porcentaje significativo; pero si evaluamos la matriz de intereses respecto a lo que pudiera explotarse y aprovecharse, sería muy interesante estructurar una estrategia que permita aprovechar todo lo que puede representar para Colombia y para sus sectores económicos la generación de empleo y la transferencia de tecnología y buenas prácticas de manufactura.

Por otra parte, la cooperación internacional puede convertirse en ese complemento que requieren los sectores económicos de Colombia, en términos de asociatividad, mecanismos que eleven la competitividad, etc.

Colombia puede lograr bilateralmente lo que no ha podido multilateralmente, lo que a pesar de ser una excepción a la regla en términos de negociación internacional, puede facilitar el proceso y posibilitar el aprovechamiento de nuevos espacios de integración, desde la transferencia de tecnología y conocimiento, hasta las buenas prácticas agrícolas, de manufactura y educación. Igualmente, este proceso se convierte en una invitación a todos los actores para se atrevan a buscar nuevas maneras de relacionamiento y de complementación en las distintas formas y constructos sociales, y que no pierdan de vista que la integración es, ante todo, para mejorar las condiciones existentes de las personas.

La estrategia de inserción internacional de Colombia al Foro de Cooperación Económica Asia Pacífico y fortalecer sus relaciones comerciales con China puede ser su logro más grande y la posibilidad de gestionar nuevas formas de cooperación e integración. Es ente sentido, es fundamental que el país diseñe políticas exteriores más reales y menos utópicas, que generen respuestas y disminuyan las preguntas para el bien de todos. 
LIINEA DE INVESTIGACIÓN: DESARROLLO ECONÓMICO, SECTORIAL E INDUSTRIAL

\section{REFERENCIAS}

APEC. (23 de noviembre de 2016). Cumbre Cooperación Económica Asia Pacífico APEC . Recuperado de http://www.apec2016.pe/es/

Ardila, M. (2005). Colombia y su política exterior en el siglo XXI. Bogotá: Friedrich Ebert Stiftung en Colombia.

DANE. (25 de noveimbre de 2016). Exportaciones. Recuperado de https://www.dane.gov.co/index. php/estadisticas-por-tema/comercio-internacional/exportaciones

Hamburger, F. (2014). Santos: de hijo de la seguridad a promotor de la paz . Estudios de política exterior, (158), 68-76.

Hernández, L. (2009). Posmodernismo y constructivismo: su utilidad para analizar la política exterior colombiana. OASIS: Observatorio de Análisis de los Sistemas Internacionales, (14), 201-218.

Ruiz, A. (25 de noviembre de 2016). La balanza comercial de Colombia con China sigue siendo deficitaria. La República. Recuperado de http://www.larepublica.co/la-balanza-comercial-decolombia-con-china-sigue-siendo-deficitaria_257251

Lamus, F. (2015). El soft power chino. Un acercamiento. Revista Enfoques, 9(14), 21-51.

Mateus, L. (2015). Inserción de Colombia en el mercado de China, hacia un tratado de libre comercio Colombia-China (tesis de pregrado). Universidad del Rosario, Administración de Negocios Internacionales, Bogotá, Colombia.

Merke, F. (2007). Identidad y Política Exterior en la Teoria de las Relaciones internacionales. Buenos Aires: IDICSO, Instituto de Investigación en Ciencias Sociales, Universidad del Salvador.

Ministerio de Comercio, Industria y Turismo. (2016). Colombia: estadísticas de comercio exterior. Recuperado de http://www.mincit.gov.co/loader.php?IServicio=Documentos\&IFuncion=verPdf \&id=56116\&name=2011-ImpoAbril-presentacion.pdf\&prefijo=file

Peña, G.(2014). La política económica exterior de Colombia 2000-2014: un balance preliminar del comercio exterior y la inversión extranjera directa. Revista Análisis Internacional, 5(2), 297-313.

Piedrahita, P.(2015). Oportunidades y amenazas para Colombia de pertenecer a la Alianza del Pacífico. Bogotá: Universidad del Rosario.

Ramírez, S. (2011). El giro de la política exterior colombiana. Nueva Sociedad, 231, 79-95.

Ripoll de Castro, A. (2015). La diplomacia catalítica y la cooperación internacional descentralizada en pro de una eficaz seguridad humana: estudio de caso Colombia 2012. Bogotá: Universidad Militar Nueva Granada. 
Pérez, A., y Pérez, C. (2011). Desafíos de Colombia en APEC. Mundo Asia Pacífico, 1(1)39-50.

Sánchez, G. (2015). Colombia en la integración regional de la Alianza del Pacifico. Bogotá: Universidad Militar Nueva Granada.

Tassara, C. (2016). Colombia en el escenario internacional. Revista Internacional de Cooperación y Desarrollo, 2(2), i-ix.

Tassara, C. (2016). La política exterior de Colombia, la OCDE y la revisión entre pares de las políticas públicas. Revista Internacional de Cooperación y Desarrollo, 2(2), 69-104.

Torres, C. (2014). Hacia una política exterior colombiana sostenida en el tiempo. Revista Análisis Internacional, 5(2), 335-350.

Tremolada, E. (2015). ¿La Alianza del Pacífico facilita la inserción de Colombia en la región Asia-Pacífico? Papel Político, (julio-diciembre), 721-752.

Vidal, P. Peláez, J., y de Miranda, M. (2015). Restricciones de balanza de pagos en Colombia. Efectos de la apertura internacional y el aumento del comercio con Asia [Balance of Payment Restrictions in Colombia. Effects of International Liberalization and Increase of Trade with Asia]. Cali: Pontificia Universidad Javeriana.

Villa, C. (2015). La proyección de Colombia hacia el Asia-Pacífico en un contexto de posacuerdo. En E. Pastrana y H. Buelvas (eds.), Política Exterior Colombiana Escenarios y Desafíos en el Posconflicto (pp. 567-594). Bogotá: Pontificia Universidad Javeriana. 